\title{
Determining Risk of Vertebral Osteoporosis in Men: Validation of the Male Osteoporosis Risk Estimation Score
}

\author{
Angela J. Shepherd, MD, Alvah R. Cass, MD, SM, and Laura Ray, MA
}

Background: Vertebral fracture, one of the most frequent osteoporotic fractures in both sexes, is a powerful indicator of future osteoporotic fractures. Vertebral fractures are associated with increased mortality and decreased quality of life. Osteoporosis is a major predictor of low-trauma fracture. The Male Osteoporosis Risk Estimation Score (MORES), a clinical prediction tool that uses age, weight, and a history of chronic obstructive pulmonary disorder, was developed and validated previously to identify men at risk for hip osteoporosis who might benefit from bone densitometry. This study evaluated the effectiveness of the MORES to identify men at risk of lumbar osteoporosis.

Methods: US population data from the National Health and Nutrition Examination Survey (NHANES, 1999-2004) were used to test the validity of the MORES to identify men at risk of lumbar osteoporosis.

Results: The MORES value was compared with vertebral done densitometry (DXA) scores for men 50 years of age and older. The sensitivity was 0.582 (95\% CI, 0.460-0.694) and specificity was 0.652 (95\% CI, 0.627-0.676). Comparing universal DXA screening in 50-year-old men, the number needed to screen (NNS) to prevent one case of vertebral fracture would be reduced from 9418 to 3641 by prescreening with the MORES. In 70-year-old men, the MORES reduced the NNS from 4987 with universal screening to 3583 .

Conclusion: This analysis validated the MORES as a clinical tool to identify men at risk for lumbar osteoporosis. Compared with universal screening, the MORES was able to reduce the NNS to prevent one additional vertebral fracture across all age groups except in men 85 to 89 years of age. The magnitude of the NNS to prevent one additional vertebral fracture does not support using the MORES to screen solely for osteoporosis of the lumbar spine. (J Am Board Fam Med 2010;23:186-194.)

Keywords: Osteoporosis, Men, Osteoporosis Risk Estimates, Validation

The prevalence of osteoporosis in men is expected to increase by nearly $50 \%$ during the next 15 years. ${ }^{1}$ Worldwide, hip fracture rates are expected to double by 2025 , with a disproportionate increase in men. ${ }^{2}$ Osteoporosis is a major predictor of future fractures; therefore, the need to understand risk factors for osteoporosis and osteoporotic fractures

This article was externally peer reviewed.

Submitted 13 February 2009; revised 29 July 2009; accepted 6 August 2009.

From the Department of Family Medicine (AJS, ARC) and the Department of Preventive Medicine and Community Health (LR), University of Texas Medical Branch, Galveston.

Funding: Joint American Academy of Family Physicians/ Fellow of American Academy of Family Physicians Grant Awards Program (G0509).

Conflict of interest: none declared.

Corresponding author: Angela J. Shepherd, MD, Associate Professor, Department of Family Medicine, University of Texas Medical Branch, 301 University Boulevard, Galveston, TX 77555 (E-mail: ajshephe@utmb.edu). in men deserves immediate attention. ${ }^{3}$ We previously reported on the development and initial validation of the Male Osteoporosis Risk Estimation Score (MORES), ${ }^{4}$ a clinical instrument that effectively identified men at increased risk of osteoporosis of the hip; however, the ability to predict vertebral osteoporosis was not addressed.

Vertebral fractures are the most common osteoporotic fracture found in men and women ${ }^{5,6}$ and are a strong predictor of future osteoporotic fractures. ${ }^{7-11}$ Like hip fractures, vertebral osteoporotic fractures are associated with significant mortality and morbidity. ${ }^{12-15}$ Vertebral fractures in men have been associated with decreased survival when compared with men who do not fracture, and with greater mortality risk when compared with women with vertebral fractures. ${ }^{15}$ Vertebral fractures, and the resultant increase in mortality and morbidity, are not inevitable. Estimates of reduction in fracture risk for men who re- 
ceive treatment for osteoporosis are similar to those achieved by women receiving treatment. ${ }^{16-22}$ In addition, based on a recent meta-analysis, treatment of osteoporosis with alendronate produced a greater reduction of risk for vertebral fractures (0.52) than the risk reduction observed with treatment of osteoporosis of the hip (0.63). ${ }^{20}$

Compounding the expected increase in osteoporotic fractures are reports indicating that osteoporosis among men is still substantially underdiagnosed, undertreated, and inadequately researched. ${ }^{1,23}$ In 2007 the International Society for Clinical Densitometry addressed the underdiagnosis issue with a recommendation for universal bone mineral density (BMD) screening for men 70 years of age and older. ${ }^{24,25}$ In 2008, the National Osteoporosis Foundation included men in their screening recommendations, advocating BMD testing for men 50 to 70 years of age who have one or more risk factors for osteoporosis and BMD testing of men at age $\geq 70$ years without risk factors. ${ }^{26}$ These recommendations emphasize the importance of screening but they lack specificity and therefore may not be applied uniformly or efficiently in clinical practice.

Recently published studies have identified specific risk factors for osteoporosis in men. The Osteoporosis Fractures in Men (MrOS), a large observational study, found that factors such as age, race, height, change in height, personal and family history of fracture, diagnoses of chronic obstructive pulmonary disorder (COPD) or kidney stones, and the use of selective serotonin reuptake inhibitors predicted BMD.$^{27}$ However, MrOS did not address application of the findings in a clinical setting. In a systematic review Espallargues and colleagues ${ }^{28}$ stratified an extensive list of risk factors into 3 categories: high risk for fracture, moderate risk for fracture, and no risk or protective fracture. They suggest that these factors can be used to guide BMD testing decisions. More recently, the MORES used age, low body weight $(<70 \mathrm{~kg})$, and a history of COPD to effectively identify men with an increased risk of osteoporosis of the hip. ${ }^{4}$ The MORES is a simple instrument that can be readily applied in the clinical encounter to select men for screening for osteoporosis of the hip; however, the MORES has not been evaluated for identifying osteoporosis of the lumbar spine.

The primary objective of this study was to evaluate the clinical performance of the MORES to identify men at risk for lumbar osteoporosis and to assess the effectiveness of screening men for lumbar osteoporosis. In addition, we conducted a stratified analysis of effectiveness across 3 ethnic groups.

\section{Methods \\ Subjects}

Participants eligible for this study included 2984 men aged 50 years and older who had been included in any of the National Health and Nutrition Examination Survey (NHANES) 1999 to 2000, 2001 to 2002, and 2003 to 2004 datasets and who had a valid whole-body dual-energy radiograph absorptiometry (DXA) scan. Forty subjects were eliminated from the analysis because of missing values for variables essential for a weighted analysis (35 non-Hispanic white men and 5 men of unspecified race/ethnicity). The NHANES datasets from the 1999 to 2004 surveys represent a national probability sample of the civilian, noninstitutionalized US population. ${ }^{29}$ The surveys were conducted by the National Center for Health Statistics and Centers for Disease Control and Prevention. Details of the sampling and data collection have been described elsewhere. ${ }^{29}$ The DXA scans were done using a Hologic QDR-4500A fan beam densitometer (Hologic, Inc., Bedford, Massachusetts). Hologic software version 8.26:a $3^{*}$ was used to administer all scans.

Men were excluded from DXA examination because of self-reported history of radiographic contrast material (barium) use during the previous 7 days, self-reported nuclear medicine studies during the previous 3 days, and self-reported weight more than 300 pounds or height more than 6'5" (DXA table limitations). ${ }^{30}$ The study protocol was approved by the Institutional Review Board of the University of Texas Medical Branch.

\section{Data and Variables}

Inclusion criteria were male sex and age of $\geq 50$ years with a readable DXA. Variables included (1) race/ethnicity (self-identified) of white, black, Mexican-American, or "other" (other Hispanic, other racial groups including a multiracial designation, and those missing a specific response to race $)^{29} ;(2)$ measured weight; and (3) the presence of COPD (self-reported emphysema or chronic bronchitis). Age was modeled as an ordinal variable, and the youngest age group served as the reference group. Weight was modeled as an ordinal variable with the 
Table 1. Male Osteoporosis Risk Estimation Score ${ }^{4}$

\begin{tabular}{lc}
\hline Risk Factor & MORES Points* \\
\hline Age (years) & \\
$\quad \leq 55$ & 0 \\
$56-74$ & 3 \\
$\quad \geq 75$ & 4 \\
Weight, kg (lbs) & \\
$\quad \leq 70(\leq 154)$ & 6 \\
$>70-80 \mathrm{~kg}(>154-176)$ & 4 \\
$>80(>176)$ & 0 \\
COPD & 3
\end{tabular}

*Screening threshold is $\geq 6$ points.

MORES, Male Osteoporosis Risk Estimation Score; COPD, chronic obstructive pulmonary disorder.

heaviest weight being the reference group. History of COPD was modeled as a dichotomous variable based on self-report, and men with no history of COPD served as the reference group. Point assignments for each of these groups were the same as those used in the original validation of the MORES index. $^{4}$

\section{MORES}

The MORES ${ }^{4}$ is a clinical prediction rule designed for use in an office setting to select men for DXA screening of the hip. The instrument was developed and validated in the NHANES 1994 to 1998 (NHANES III) and is defined by 3 factors: age, weight, and history of COPD. The MORES is a weighted scale developed from a logistic regression model using gender-neutral risk factors for osteoporosis or osteoporosis-related fracture and was validated using split sample methods. Table 1 lists the variables, scoring system, and threshold values assigned for the MORES. Predictor variables evaluated during development included sociodemographic data (age, weight, race/ethnicity, level of education, and marital status); medical and family history (medical comorbidities, family history of osteoporosis, and maternal history of hip fracture); health-related behaviors (use of alcohol and tobacco and level of physical activity); and self-rated health status. For osteoporosis of the hip, the MORES had an overall sensitivity of 0.93 (95\% CI, $0.85-0.97$ ) and a specificity of 0.59 (95\% CI, 0.560.62 ) with an estimated area under the curve (AUC) of 0.832 (95\% CI, 0807-0.858). In a number needed to screen (NNS) analysis, the MORES reduced the NNS to prevent one additional hip frac- ture by approximately 50\% across all age groups compared with universal screening. ${ }^{4}$

Osteoporosis at each site was defined as a BMD $\leq 2.5$ SD below the mean, using white men 20 to 29 years of age as the reference group, in accordance with the World Health Organization criteria. ${ }^{31}$

\section{Analysis}

Using $2 \times 2$ contingency tables we estimated the sensitivity and specificity of the MORES using the previously determined cut score of $\geq 6$ for predicting osteoporosis of the lumbar vertebra and for any measured site (thoracic vertebra, lumbar vertebra, arms, ribs, pelvis, or legs). Skull data were not included in this study. AUC for the MORES was also estimated. Analyses were performed using statistical analysis software 9.2 with SUDAAN 9.1.3 (RTI International, Research Triangle Park, NC) to adjust for design effects and weighted sampling. ${ }^{32,33}$ Imputed data provided by NHANES 1999 to 2004 was not included in any of the analyses. To determine the clinical usefulness of the MORES we constructed a table comparing the NNS to prevent one additional vertebral fracture during the next 5 years for successive age categories of men. We constructed the table following the method described by Nelson et $\mathrm{al}^{34}$ to develop the US Preventive Services Task Force (USPSTF) recommendations for screening for osteoporosis in women. Age-specific prevalence of osteoporosis was derived from the NHANES 1999 to 2004 data. ${ }^{30}$ To allow comparison with results of the USPSTF analysis, which used age-group-specific, 5 -year fracture rates, we estimated age-specific, 5 -year vertebral fracture rates for men from data published by Kanis et $\mathrm{al}^{35}$ by halving the mean 10 -year fracture risk for each 5-year age category. Assumptions for risk reduction with treatment and adherence to treatment were based on the best available information from the medical literature and duplicated the assumptions used by Nelson et $\mathrm{al}^{34}{ }^{34}$ where appropriate. Formulas for calculation are available by request. Where appropriate, results were reported by race/ethnicity to illustrate the effect of race/ethnicity on the operating characteristics of the MORES.

\section{Results}

The study population included a total of 2944 men $\geq 50$ years of age who had an interpretable DXA. 
Table 2. Sample Demographics of Men 50 Years of Age and Older from the National Health and Nutrition Examination Survey, 1999 to 2004

\begin{tabular}{|c|c|c|}
\hline & $\mathrm{N}$ & Adjusted \% \\
\hline \multicolumn{3}{|l|}{ Race $(\mathrm{n}=2944)$} \\
\hline White & 1720 & 81.0 \\
\hline African-American & 483 & 8.2 \\
\hline Mexican-American & 573 & 3.6 \\
\hline Other & 168 & 7.2 \\
\hline \multicolumn{3}{|l|}{ Age $(n=2944)$} \\
\hline Mean & 62.99 & $95 \%$ CI, $62.53-63.44$ \\
\hline$\leq 55$ & 526 & 31.0 \\
\hline $56-74$ & 1643 & 52.9 \\
\hline$\geq 75$ & 775 & 16.1 \\
\hline \multicolumn{3}{|l|}{ Weight $(\mathrm{n}=2932)$} \\
\hline Mean & 86.12 & $95 \%$ CI, $85.35-86.87$ \\
\hline$\leq 70 \mathrm{~kg}$ & 591 & 15.2 \\
\hline $71-80 \mathrm{~kg}$ & 762 & 23.5 \\
\hline$>80$ & 1591 & 61.3 \\
\hline \multicolumn{3}{|c|}{ Osteoporosis (any site) $(\mathrm{n}=2944)$} \\
\hline No & 2580 & 89.7 \\
\hline Yes & 364 & 10.3 \\
\hline \multicolumn{3}{|c|}{ Osteoporosis (vertebral) $(\mathrm{n}=2944)$} \\
\hline No & 2818 & 95.7 \\
\hline Yes & 126 & 4.3 \\
\hline \multicolumn{3}{|l|}{$\operatorname{COPD}(\mathrm{n}=2943)$} \\
\hline No & 2708 & 92.3 \\
\hline Yes & 235 & 7.7 \\
\hline
\end{tabular}

COPD, chronic obstructive pulmonary disorder.

Based on self-report, $81 \%$ identified themselves as non-Hispanic white; $8.2 \%$ as African-American; $3.6 \%$ as Mexican-American; and $7.2 \%$ as "other" (other Hispanic, other racial groups including a
Table 3. Sample Demographics of Men by Osteoporosis Prevalence by Race/Ethnicity from the National Health and Nutrition Examination Survey, 1999 to 2004

\begin{tabular}{lcl}
\hline Race/Ethnicity & Any Site $^{*}$ & Vertebra \\
\hline White & $10.04(8.59-11.70)$ & $4.10(3.14-5.32)$ \\
African-American & $4.30(2.62-6.97)$ & $1.34(0.55-3.23)$ \\
Mexican-American & $14.46(11.03-18.72)$ & $7.22(4.66-11.03)$ \\
Other & $17.74(12.59-24.41)$ & $9.10(4.67-16.98)$ \\
Totals & $10.28(9.03-11.69)$ & $4.34(3.48-5.41)$ \\
\hline
\end{tabular}

Data provided as \% (range).

${ }^{*}$ Excludes skull.

multiracial designation, and those missing a specific response to race). Mean age was 63 years; mean body weight was $86 \mathrm{~kg}$ (189 lbs); and prevalence of COPD was $7.7 \%$. The prevalence of osteoporosis in men at any site was $10.3 \%$ (95\% CI, 9.0-11.7) and the prevalence of vertebral osteoporosis was $4.3 \%$ (95\% CI, 3.5-5.4). Results are reported in Table 2. ${ }^{30}$ The prevalence of osteoporosis among African-American men $(n=483)$ was lower than for other racial/ethnic groups, as reported in Table $3 .{ }^{30}$

The sensitivity for the MORES was 0.655 (95\% CI, 0.582-0.721) for identifying men with osteoporosis at any site; the specificity was 0.675 (95\% CI, 0.649-0.701); and AUC was 0.728. When limiting the area of interest to the lumbar spine, the sensitivity for men was 0.582 (95\% CI, 0.460 $0.694)$ and specificity was 0.652 (95\% CI, $0.627-$ $0.676)$; and AUC was 0.657. Complete results stratified by race/ethnicity are reported in Table 4.

Table 4. Operating Characteristics of the Male Osteoporosis Risk Estimation Score in Predicting Osteoporosis

\begin{tabular}{|c|c|c|c|c|c|c|}
\hline & \multicolumn{6}{|c|}{ Osteoporosis } \\
\hline & \multicolumn{3}{|c|}{ Any site } & \multicolumn{3}{|c|}{ L-Vertebra } \\
\hline & Sensitivity* (95\% CI) & Specificity* $(95 \%$ CI $)$ & $\mathrm{AUC}^{*}$ & Sensitivity* $\left.^{*} 95 \% \mathrm{CI}\right)$ & Specificity* $^{*} 95 \%$ CI $)$ & $\mathrm{AUC}^{*}$ \\
\hline $\begin{array}{l}\text { Overall (all men; } \\
\mathrm{n}=2944 \text { ) }\end{array}$ & $0.655(0.582-0.721)$ & $0.675(0.649-0.701)$ & 0.728 & $0.582(0.460-0.694)$ & $0.652(0.627-0.676)$ & 0.657 \\
\hline \multicolumn{7}{|l|}{ By Race/Ethnicity } \\
\hline White $(\mathrm{n}=1720)$ & $0.599(0.518-0.675)$ & $0.694(0.666-0.721)$ & 0.721 & $0.511(0.381-0.639)$ & $0.672(0.646-0.698)$ & 0.653 \\
\hline $\begin{array}{l}\text { African-American } \\
\quad(\mathrm{n}=483)\end{array}$ & $0.787(0.486-0.935)$ & $0.629(0.582-0.673)$ & 0.781 & $0.763(0.253-0.969)$ & $0.616(0.566-0.664)$ & 0.786 \\
\hline $\begin{array}{l}\text { Mexican-American } \\
\quad(\mathrm{n}=573)\end{array}$ & $0.713(0.578-0.819)$ & $0.588(0.528-0.645)$ & 0.703 & $0.596(0.395-0.768)$ & $0.555(0.499-0.610)$ & 0.601 \\
\hline Other $(\mathrm{n}=168)$ & $0.951(0.825-0.987)$ & $0.551(0.449-0.650)$ & 0.675 & $0.904(0.662-0.978)$ & $0.499(0.402-0.596)$ & 0.648 \\
\hline
\end{tabular}

*All values were adjusted for sampling and design effects using SUDAAN software.

AUC, area under the curve. 
Table 5. Screening for Vertebral Osteoporosis in 10,000 Men $\geq 50$ Years of Age: Vertebral Fracture Outcomes by 5-Year Intervals

\begin{tabular}{|c|c|c|c|c|c|c|c|c|}
\hline Age Group (years) & $50-54$ & $55-59$ & $60-64$ & $65-69$ & $70-74$ & $75-79$ & $80-84$ & $85-89$ \\
\hline Sensitivity & 0.288 & 0.467 & 0.878 & 0.823 & 0.71 & 0.839 & 0.851 & 0.751 \\
\hline Specificity & 0.898 & 0.637 & 0.671 & 0.618 & 0.497 & 0.472 & 0.31 & 0.227 \\
\hline \multicolumn{9}{|l|}{ Base/Case Assumptions per 10,000} \\
\hline 5 -yr risk of vertebral fracture ${ }^{* 35}$ & 0.006 & 0.008 & 0.010 & 0.013 & 0.017 & 0.021 & 0.021 & 0.014 \\
\hline Osteoporosis risk ${ }^{30}$ & 0.050 & 0.051 & 0.039 & 0.026 & 0.035 & 0.051 & 0.055 & 0.025 \\
\hline $\begin{array}{l}\text { Relative risk for vertebra fracture } \\
\mathrm{w} / \mathrm{Rx}^{34}\end{array}$ & 0.52 & 0.52 & 0.52 & 0.52 & 0.52 & 0.52 & 0.52 & 0.52 \\
\hline Adherence to $\mathrm{Rx}$, proportion ${ }^{34}$ & 0.7 & 0.7 & 0.7 & 0.7 & 0.7 & 0.7 & 0.7 & 0.7 \\
\hline \multicolumn{9}{|l|}{ Results, per 10,000 men (n) } \\
\hline Predicted cases of osteoporosis (n) & 501.6 & 513.1 & 394.2 & 263.8 & 345.0 & 508.6 & 549.7 & 248.3 \\
\hline MORES TP & 144.5 & 239.6 & 346.1 & 217.1 & 245.0 & 426.7 & 467.8 & 186.5 \\
\hline MORES FN & 357.2 & 273.5 & 48.1 & 46.7 & 100.1 & 81.9 & 81.9 & 61.8 \\
\hline MORES TN & 8529.5 & 6043.1 & 6445.5 & 6017.0 & 4798.5 & 4480.0 & 2929.6 & 2213.6 \\
\hline MORES FP & 968.8 & 3443.7 & 3160.3 & 3719.2 & 4856.5 & 5011.5 & 6520.7 & 7538.1 \\
\hline Referred for DXA (TP + FP) & 1113.3 & 3683.4 & 3506.4 & 3936.3 & 5101.4 & 5438.2 & 6988.5 & 7724.5 \\
\hline \multicolumn{9}{|l|}{$\begin{array}{l}\text { Predicted Vertebral Fx: MORES/ } \\
\text { DXA screening (n) }\end{array}$} \\
\hline TP with $\mathrm{Rx}$ & 0.60 & 1.24 & 2.18 & 1.87 & 2.81 & 5.81 & 6.52 & 1.67 \\
\hline FN with no Rx & 2.25 & 2.13 & 0.46 & 0.61 & 1.73 & 1.68 & 1.72 & 0.83 \\
\hline Total $F_{x}$ with $\mathrm{Rx}$ after screening & 2.85 & 3.37 & 2.64 & 2.48 & 4.54 & 7.49 & 8.24 & 2.51 \\
\hline Unscreened, untreated & 3.16 & 4.00 & 3.74 & 3.43 & 5.97 & 10.43 & 11.54 & 3.35 \\
\hline NNS MORES: DXA & 3641 & 5866 & 3175 & 4151 & 3583 & 1851 & 2118 & 9134 \\
\hline NNT MORES: DXA & 1641 & 818 & 357 & 279 & 243 & 174 & 167 & 294 \\
\hline \multicolumn{9}{|l|}{ Universal DXA screening (n) } \\
\hline Fracture with $\mathrm{Rx}$ & 2.10 & 2.66 & 2.49 & 2.28 & 3.96 & 6.92 & 7.66 & 2.23 \\
\hline Fracture with no $\mathrm{Rx}$ & 3.16 & 4.00 & 3.74 & 3.43 & 5.97 & 10.43 & 11.54 & 3.35 \\
\hline NNS universal DXA & 9418 & 7437 & 7949 & 8678 & 4987 & 2855 & 2579 & 8880 \\
\hline NNT universal DXA & 473 & 382 & 314 & 229 & 173 & 146 & 142 & 221 \\
\hline
\end{tabular}

*Mean, 10 years risk across age groups $\div 2$.

MORES, Male Osteoporosis Risk Estimation Score; Rx, bisphosphonate therapy; TP, true positives; FN, false negatives; TN, true negatives; FP, false positives; DXA, done densitometry; NNS, number needed to screen to prevent one additional vertebral fracture; NNT, number needed to treat to prevent one additional vertebral fracture; Fx, fracture.

We constructed an NNS table to evaluate the impact of the MORES on screening for lumbar osteoporosis. The MORES, when used as a preDXA screening tool, consistently reduced the NNS compared with universal screening except in the 85 - to 89-year-old age group. For example, the MORES produced NSS in men 50 to 69 years of age (3641-5866) that were similar to the NNS for universal screening in men 70 to 74 years of age (4987), the age recommended for universal screening by the International Society for Clinical Densitometry $^{24,25}$ and the National Osteoporosis Foundation. ${ }^{26}$ Full results are reported in Table 5.

\section{Discussion}

In this independent sample of men represented in the NHANES 1999 to 2004 datasets, the MORES proved to be a valid instrument that can be used to identify a subgroup of men at increased risk of osteoporosis at the lumbar spine as well as other combined sites. The operating characteristics of the MORES demonstrated minor differences across racial/ethnic groups. Compared with universal screening, the MORES was able to reduce the NNS to prevent one additional vertebral fracture across all age groups, except in men 85 to 89 years of age. However, the NNS to prevent one additional vertebral fracture using the MORES is greater than the NNS to prevent one additional hip fracture. The magnitude of the NNS to prevent one additional vertebral fracture may not support using the MORES to screen solely for osteoporosis of the lumbar spine. 
The NNS to prevent one additional fracture at any given site is dependent on a series of complex interactions between the prevalence of osteoporosis, operating characteristics of the MORES, the rate of osteoporotic fractures, and the effectiveness of therapy. Each of these points is addressed because it may have influenced the results observed in this study.

\section{Prevalence}

Among men, there is a lower prevalence of DXAdefined osteoporosis at the lumbar vertebra compared with hip. ${ }^{36}$ In addition, the prevalence potentially may be affected by concurrent osteoarthrosis. Osteoarthrosis involves hypertrophy of the bone and increases apparent BMD without necessarily increasing strength, an observation also reported by Cauley et $\mathrm{al}^{27}$ in the MrOS Research Group report and by Orwoll et al. ${ }^{37}$ The competing effects of the expected decline in BMD associated with aging and the expected increase in BMD associated with osteoarthrosis over time may lead to misclassification of men with lumbar osteoporosis as "normal," which may render densitometry less reliable for the diagnosis of lumbar osteoporosis. In effect, this misclassification decreases the observed prevalence of osteoporosis and necessarily increases the NNS to prevent one additional vertebral fracture at any given age.

\section{Operating Characteristics}

In our previous work ${ }^{4}$ we demonstrated that the MORES is an effective and valid instrument that can be used to identify men at risk for osteoporosis of the hip with relatively high sensitivity and moderate specificity. In the current study, we demonstrated that the MORES is a valid instrument that can be used to identify men at risk for osteoporosis of the lumbar spine; however, the observed decrease in sensitivity resulted in poorer discrimination for lumbar osteoporosis compared with hip. (See Table 4 for details.) The potential misclassification of osteoporosis, because of the effect of osteoarthrosis on BMD discussed previously, may influence the operating characteristics of the MORES. For example, the MORES relies heavily on age as a risk factor for osteoporosis. Assuming osteoarthrosis also increases with age, patients who meet the screening threshold on the MORES because of the influence of age may be misclassified as having a "normal" BMD with DXA because of the effect of osteoarthrosis, which would probably result in a loss of sensitivity. Another explanation for the reduced sensitivity of the MORES could be that risk factors other than age, weight, and COPD (used to predict osteoporosis of the hip) may be better predictors of vertebral osteoporosis. In either event, the reduction in sensitivity resulted in an increase in the NNS to prevent one additional vertebral fracture.

\section{Event Rates}

In general, we have more specific and more precise data available for osteoporosis of the hip and hip fractures than for vertebral osteoporosis and related fractures. In the original MORES study (hip) we were able to use hip fracture rates given a BMD consistent with osteoporosis, ie, $\mathrm{t}$ score less than -2.5. However, in the current analysis we were unable to find published data with comparable specificity and precision. Therefore, we used the best data available and estimated vertebral fracture rates without respect to $\mathrm{BMD}$ or site of fracture (thoracic vs lumbar). This resulted in a lower fracture event rate than one might expect given osteoporosis of the spine and therefore would effectively increase the NNS to prevent one additional vertebral fracture. In addition, age-related fractures of the spine increase at a lesser rate than hip fractures. Therefore, as age increases, fracture rates for the spine have less effect on reducing the NNS to prevent one additional fracture than do fracture rates for the hip.

\section{Effectiveness}

The increased effectiveness of treatment resulting in the prevention of vertebral fracture compared with hip fracture should decrease the NNS to prevent one additional vertebral fracture. The relative risk for vertebral fracture with treatment is reported as 0.52 versus the relative risk for hip fracture with treatment of $0.63 .{ }^{34}$ However, the opposing effects of the other 3 factors described above seem to override the effectiveness of treatment.

An existent vertebral fracture is a strong predictor for a future fracture at any site, but the predictive value of the vertebral BMD may be of less clinical importance. In addition, although a low vertebral BMD accurately predicts osteoporosis and an increased risk of vertebral fracture, a normal vertebral BMD may not exclude osteoporosis and subsequent risk of fracture because of coexistent osteoarthrosis. Newer modalities, such as the inclu- 
sion of a vertebral fracture analysis, done at the same time as the bone density measurement, may provide more clinically pertinent information regarding the integrity of the vertebral bodies.

\section{Limitations}

Our study had several limitations. First, the lack of reported BMD measurements of the hip in the NHANES 1999 to 2004 datasets prevented validation of the MORES for selecting men with an increased risk of osteoporosis of the hip alone or in combination with the lumbar spine. Simultaneous consideration of BMD measurements of the hip and lumbar spine is the usual approach taken during the clinical encounter. Second, the data related to the probability of experiencing a future vertebral fracture are less specific and less precise than are the data regarding the probability of a future hip fracture. Third, the NNS analysis depended on several assumptions regarding effectiveness of, and adherence to, treatment. Although these assumptions and estimates are derived from clinical trial data and meta-analyses, they may be optimistic when applied to clinical practice, a point noted by Nelson et $\mathrm{al}^{34}$ in the USPSTF recommendations for women. Finally, the NNS analysis is a surrogate measure for the impact of screening for osteoporosis as opposed to the measurement of hard endpoints such as actual fractures and fracture-related morbidity and mortality.

\section{Future Studies}

Further evaluation of the MORES in a clinical setting is warranted to assess the effectiveness of the MORES as a prescreening tool for osteoporosis at the hip alone and combined with osteoporosis of the spine. Validation of the MORES for vertebral osteoporosis, which was the primary focus of this study, proved to be more complex because of the effects of osteoarthrosis on BMD. Vertebral fracture analysis, which is now available on many densitometers, is a promising addition to evaluating the risk of fractures. ${ }^{38-40}$ Vertebral fracture analysis measures height differences between the anterior and posterior segments of each vertebra and may be a better predictor of the risk of vertebral osteoporosis and fracture. Vertebral fracture analysis may overcome misclassification of osteoporosis because of coexistent osteoarthrosis. We plan clinical studies to evaluate the effectiveness of the MORES to identify men at risk for hip and/or vertebral osteo- porosis/fracture using a combination of BMD and vertebral fracture analysis.

Finally, studies examining barriers to osteoporosis screening and treatment by clinicians, both in the hospital and ambulatory setting, are also necessary. Ultimately the effectiveness of a clinical prediction rule hinges on changing clinical behavior. Changing clinical behavior is a daunting challenge, which is illustrated by the fact that $<20 \%$ of patients receive therapeutic interventions for osteoporosis even after suffering an osteoporotic fracture. $^{23,41,42}$

\section{Conclusion}

The current analysis validated the MORES as a clinical tool capable of identifying men at an increased risk for lumbar osteoporosis. Furthermore, the MORES performed similarly across racial/ethnic groups. The MORES did not discriminate as well for lumbar osteoporosis (AUC, 0.657) when compared with osteoporosis of the hip (AUC, 0.832). ${ }^{4}$ Compared with universal screening the MORES reduced the NNS to prevent one additional vertebral fracture in all age groups, except in men 85 to 89 years of age. However, the reduction in NNS may be insufficient to support using the MORES to screen solely for osteoporosis of the lumbar spine.

Proven strategies are available to reduce the impact of osteoporosis. Translating these strategies into improved patient outcomes depend on the integration of all levels of health care providers and health care delivery systems. For primary care clinicians, who carry the mantle for comprehensive patient care regardless of setting, it is imperative that we advocate for patients, collaborate with others involved in the care of patients with osteoporosis and osteoporotic fractures, and work in all settings to shift the prevention and treatment of osteoporosis and related fractures from an afterthought to the forefront of care. Future trends in early recognition and treatment of osteoporosis to reduce osteoporotic fractures will be the ultimate measure of our success.

The authors wish to acknowledge Carol Carlson, BA.

\section{References}

1. Looker A, Orwoll E, Johnston C, et al. Prevalence of low femoral bone density in older US adults from NHANES III. J Bone Miner Res 1997;12:1761-8. 
2. Gullberg B, Johnell O, Kanis J. World-wide projections for hip fracture. Osteoporos Int 1997;7:407-13.

3. Orwoll E, Blank J, Barrett-Connor E, et al. Design and baseline characteristics of the osteoporotic fractures in men (MrOS) study: a large observational study of the determinants of fracture in older men. Contemp Clin Trials 2005;26:569-85.

4. Shepherd A, Cass A, Carlson C, Ray L. Development and internal validation of the Male Osteoporosis Risk Estimation Score. Ann Fam Med 2007;5: 540-6.

5. O’Neill T, Felsenberg D, Varlow J, Cooper C, Kanis J, Silman A. The prevalence of vertebral deformity in European men and women: the European Vertebral Osteoporosis Study. J Bone Miner Res 1996;11:1010-8.

6. Naves-Diaz M, Diaz-Lopez J, Gomez-Alonso C, Altadill-Arregui A, Rodriguez-Rebollar A, CannataAndia J. Study of incidence of osteoporotic fractures in a cohort of individuals older than 50 years from Asturias, Spain, after a 6 year follow-up period. Med Clin (Barc) 2000;115:650-3.

7. Bernstein D, Sadowsky N, Hegsted D, Guri C, Stare F. Prevalence of osteoporosis in high- and low-fluoride areas in North Dakota. JAMA 1966;198:499504.

8. Lauritzen J, Lund B. Risk of hip fracture after osteoporosis fractures: 451 women with fracture of lumbar spine, olecranon, knee or ankle. Acta Orthop Scand 1993;64:297-300.

9. Kotowicz M, Melton L III, Cooper C, Atkinson E, O'Fallon W, Riggs B. Risk of hip fracture in women with vertebral fracture. J Bone Miner Res 1994;9: 599-605.

10. Gunnes M, Mellstrom D, Johnell O. How well can a previous fracture indicate a new fracture? A questionnaire study of 29802 postmenopausal women. Acta Orthop Scand 1998;69:508-12.

11. Melton L III, Atkinson E, Cooper C, O'Fallon W, Riggs B. Vertebral fractures predict subsequent fractures. Osteoporos Int 1999;10:214-21.

12. Cooper C, Atkinson E, Jacobsen S. Populationbased study of survival after osteoporotic fractures. Am J Epidemiol 1993;137:1001-5.

13. Ismail A, O’Neill T, Cooper C. Mortality associated with vertebral deformity in mena and women: results from the European Prospective Osteoporosis Study (EPOS). Osteoporos Int 1998;8:291-7.

14. Kado D, Browner W, Palermo L, Nevitt M, Genant H, Cummings S. Vertebral fractures and mortality in older women. Arch Int Med 1999;159:1215-20.

15. Lau E, Ong K, Kurtz S, Schmier J, Edidin A. Mortality following the diagnosis of a vertebral compression fracture in the medicare population. J Bone Joint Surg 2008;90:1479-86.

16. Dawson-Hughes B, Harris S, Krall E, Dallal G. Effect of calcium and vitamin D supplementation on bone density in men and women 65 years of age or older. N Engl J Med 1997;337:670-6.

17. Adachi J, Bensen W, Brown J, et al. Intermittent etidronate therapy to prevent corticosteroid-induced osteoporosis. N Engl J Med 1997;337:382-7.

18. Cohen S, Levy R, Keller M, et al. Risedronate therapy prevents corticosteroid-induced bone loss. Arthritis Rheum 1999;42:2309-18.

19. Orwoll E, Ettinger M, Weiss S, et al. Alendronate for the treatment of osteoporosis in men. N Engl J Med 2000;343:604-10.

20. Cranney A, Wells G, Willan A, et al. Meta-analysis of therapies for postmenopausal osteoporsis. II. Meta-analysis of alendronate for the treatment of postmenopausal women. Endocr Rev 2002;23:50816.

21. Ringe J, Dorst A, Faber H, Ibach K. Alendronate treatment of established primary osteoporosis in men: 3-year results of a prospective, comparative, two-arm study. Rheumatol Int 2004;24:110-3.

22. Sawka A, Papaioannou A, Adachi J, Gafni A, Hanley D, Thabane L. Does alendronate reduce the risk of fracture in men? A meta-analysis incorporating prior knowledge of anti-fracture efficacy in women. BMC Musculoskelet Disord 2005;6:39.

23. Kiebzak G, Beinart G, Perser K, Ambrose C, Siff S, Heggeness M. Undertreatment of osteoporosis in men with hip fracture. Arch Int Med 2002;162:221722.

24. Leib E, Lewiecki M, Binkley N, Hamdy R. Official positions of the International Society for Clinical Densitometry. South Med J 2004;97:107-10.

25. International Society for Clinical Densitometry. Bone Densitometry course: clinician course syllabus and associate reading materials. Lake Tahoe, CA: International Society for Clinical Densitometry; 2007.

26. National Osteoporosis Foundation. Clinician's guide to prevention and treatment of osteoporosis. Available from: http://www.nof.org/professionals/NOF_ Clinicians_Guide.pdf. Accessed 12 November 2008.

27. Cauley J, Fullman R, Stone K, et al. Factors associated with the lumbar spine and proximal femur bone mineral density in older men. Osteopor Int 2005;16: 1525-37.

28. Espallargues M, Sampietro-Colom L, Estrada S, del Rio L, Setoain J. Identifying bone-mass-related risk factors for fracture to guide bone densitometry measurements: a systematic review of the literature. Osteopor Int 2001;12:811-22.

29. National Center for Health Statistics. National Health and Nutrition Examination Survey, 2002. Available from: http://www.cdc.gov/nchs/nhanes. htm. Accessed 21 June 2006.

30. National Health and Nutrition Examination Survey: technical documentation for the 1999-2004 dualenergy X-ray absorptiometry (DXA) multiple imputation data files. Available from: http://www.cdc.gov/ 
nchs/data/nhanes/dxa/dxa_techdoc.pdf. Accessed 6 March 2008.

31. World Health Organization. Assessment of fracture risk and its application to screening for postmenopausal osteoporosis. Geneva: World Health Organization; 1994.

32. SAS Institute. SAS/STAT user's guide, version 9.1.3. Cary, NC: SAS Institute, Inc; 2006.

33. Research Triangle Institute. SUDAAN user's manual, release 9.0. Research Triangle Park, NC: Research Triangle Institute; 2007.

34. Nelson H, Helfand M, Woolf S, Allan J. Screening for postmenopausal osteoporosis: a review of the evidence for the US Preventive Services Task Force. Ann Int Med 2002;137:529-41.

35. Kanis J, Johnell O, Oden A. Long-term risk of osteoporotic fracture in Malmo. Osteopor Int 2000;11: 669-74.

36. Melton LJ III, Atkinson E, O'Connor M, O'Fallon $W$, Riggs B. Bone density and fracture risk in men. J Bone Miner Res 1998;13:1915-23.
37. Orwoll E, Bevan L, Phipps K. Determinants of bone mineral density in older men. Osteopor Int 2000;11: 815-21.

38. Lewiecki E, Laster A. Clinical review: clinical applications of vertebral fractuer assessment by dual-energy x-ray absorptiometry. J Clin Endocrinol Metab 2006;91:4215-22.

39. Middleton E, Steel S. Routine versus targeted fracture assessment for the detection of vertebral fractures. Osteopor Int 2008;19:1167-73.

40. McCloskey E, Vasireddy S, Threlkeld J, et al. Vertebral fracture assessment (VFA) with a densitometer predicts future fractures in elderly women unselected for osteoporosis. J Bone Miner Res 2008;23:1561-8.

41. Feldstein A, Nicholson G, Orwoll E, et al. The near absence of osteoporosis treatment in older men with fractures. Osteopor Int 2005;16:953-62.

42. Kamel H, Mohammad S, Tariq S, Perry HI, Morley J. Failure to diagnose and treat osteoporosis in elderly patients hospitalized with hip fracture. Am J Med 2000;109:326-8. 